\title{
Entanglement-induced state ordering under local operations
}

\author{
Mário Ziman ${ }^{1,2,3}$ and Vladimír Bužek ${ }^{1,3,4}$ \\ ${ }^{1}$ Research Center for Quantum Information, Slovak Academy of Sciences, Dúbravská cesta 9, 84511 Bratislava, Slovakia \\ ${ }^{2}$ Faculty of Informatics, Masaryk University, Botanická 68a, 60200 Brno, Czech Republic \\ ${ }^{3}$ Quniverse, Líščie údolie 116, 84104 Bratislava, Slovakia \\ ${ }^{4}$ Abteilung für Quantenphysik, Universität Ulm, 89069 Ulm, Germany
}

\begin{abstract}
We analyze how entanglement between two components of a bipartite system behaves under the action of local channels of the form $\mathcal{E} \otimes \mathcal{I}$. We show that a set of maximally entangled states is by the action of $\mathcal{E} \otimes \mathcal{I}$ transformed into the set of states that exhibit the same degree of entanglement. Moreover, this degree represents an upper bound on entanglement that is available at the output of the channel irrespective what is the input state of the composite system. We show that within this bound the the entanglement-induced state ordering is "relative" and can be changed by the action of local channels. That is, two states $\varrho_{1}^{(i n)}$ and $\varrho_{2}^{(i n)}$ such that the entanglement $E\left[\varrho_{1}^{(i n)}\right]$ of the first state is larger than the entanglement $E\left[\varrho_{2}^{(\text {in })}\right]$ of the second state are transformed into states $\varrho_{1}^{(\text {out })}$ and $\varrho_{2}^{(\text {out })}$ such that $E\left[\varrho_{2}^{(\text {out })}\right]>E\left[\varrho_{1}^{(\text {out })}\right]$.
\end{abstract}

PACS numbers: 03.67.Mn, 03.65.Ud, 03.65.Ta

The success of quantum information theory $[1,2]$ is intimately related to the phenomenon of quantum entanglement. The better we will understand properties of this purely quantum phenomenon the deeper will be our insight into the quantum realm. Even though the importance of quantum entanglement has been clearly acknowledged by founding fathers of quantum mechanics [3], the true potential of this phenomenon has been appreciated just recently with the development of quantum information science. Over last ten years many results of fundamental importance illuminating properties of quantum entanglement have been reported. In spite of all the progress, there are still many questions that are to be answered. In particular, criteria of non-separability of arbitrarily-dimensional bi-partite systems, the study of intrinsic multi-partite entanglement in composite quantum systems $[4,5]$, or the role of quantum entanglement in macroscopic systems is presently under investigation [6]. One of the problems that has attracted interest of researchers for quite some time is the issue of "proper" measures of entanglement [7].

In general, we can identify two conceptually different approaches in various attempt to define measures of entanglement. These can be named as i) the operational approach, and ii) the formal (abstract) approach. The operational approach is based on an assumption that there exists a process, or an information protocol, in which the quantum entanglement plays the role of a new resource that provides some improvement in the performance of the protocol compared to its "classical analogue". The second approach [7] is based on postulation of the desired properties that an entanglement measure has to satisfy and defines a functional with these properties (see below).

In this paper we will adopt this second approach. We will start our discussion by addressing a specific question concerning the entanglement measures. First, we will answer the question: Do local operations preserve the entanglement-induced ordering? Then we will analyze how entangled states that exhibit the same degree of entanglement are transformed under the action of local channels.

Let us start with a trivial observation: States of bipartite quantum systems can be either entangled or separable (this is almost a tautological statement since the presence of entanglement is defined as an absence of the separability, and vice versa). On the other hand, the definition of entanglement is related to the non-existence of local hidden variable model for an observed statistics for a given state $\varrho^{A B}$ of a bi-partite system. R.F. Werner in his seminal work [8] has shown that such assumption restricts the entangled states to those that cannot be written as convex combinations of product states, i.e. $\varrho \neq \sum_{k} p_{k} \varrho_{k}^{A} \otimes \varrho_{k}^{B}$. The entanglement measure $E$ is a positive functional defined on the state space of a bipartite quantum system. Following Plenio and Vedral [7], let us summarize basic properties that any entanglement measure has to satisfy:

1. Sharpness: $E(\varrho)=0$ if and only if $\varrho^{A B}$ is separable.

2. Local unitary invariance: $E\left(\varrho^{A B}\right)=E\left(U_{A} \otimes\right.$ $\left.U_{B} \varrho^{A B} U_{A}^{\dagger} \otimes U_{B}^{\dagger}\right)$.

3. Convexity: $E\left(\sum_{k} p_{k} \omega_{k}^{A B}\right) \leq \sum_{k} p_{k} E\left(\omega_{k}^{A B}\right)$

4. Normalization: $E\left(\varrho^{A B}\right)=\max _{\varrho} E\left(\varrho^{A B}\right)$ if and only if $\varrho^{A B}=\left(\varrho^{A B}\right)^{2}, \operatorname{Tr}_{A} \varrho^{A B}=\operatorname{Tr}_{B} \varrho^{A B}=\frac{1}{2} I$. States with such properties are called as the maximally entangled states.

5. Non-increasing under local operations and classical communication (LOCC): A general LOCC transforms the original state $\varrho^{A B}$ into a mixture of states $\omega_{k}^{A B}$ with a probability $p_{k}$. We require that on average the entanglement cannot be increased, i.e. $\sum_{k} p_{k} E\left(\omega_{k}^{A B}\right) \leq E\left(\varrho^{A B}\right)$. Let us note that $\varrho_{k}^{A B}=\mathcal{E}_{k}^{A} \otimes \mathcal{E}_{k}^{B}\left[\varrho^{A B}\right]$. 
6. Pure state additivity: $E\left(\Phi_{1} \otimes \Phi_{2}\right)=E\left(\Phi_{1}\right)+E\left(\Phi_{2}\right)$ for all pure states $\Phi_{1}, \Phi_{2}$.

For all protocols in which quantum entanglement serves as a resource it is true that higher the degree of entanglement more efficient the application of the protocol is. However, even for a two-qubit system there exist several measures of entanglement satisfying the above properties (e.g. the concurrence [9], the relative entropy of entanglement [10], etc.) and nevertheless each of these measures might induce a different ordering on the set of states $[11,12]$. Thus, it is difficult to say "objectively" which states are more entangled. The maximally entangled states play a special role here, because the normalization property single them out independently of the particular measure.

Recently several authors [13-19] have investigated time evolution of the entanglement when a bi-partite system has been subjected to either local or global transformations (time evolution). In these studies, usually it has been investigated how a maximally entangled state is affected by the action of the corresponding evolution operation. Certainly, it is very instructive to understand what happens if the bi-partite system is initially prepared in a non-maximally entangled state $\varrho$ (in order to simplify the notation, in what follows we will omit superscripts indicating that the density operator describes two systems $A$ and $B$ ). To illuminate this problem from a perspective of an action of local channels let us formulate the following theorem.

Theorem. For each state $\omega \in \mathcal{S}(\mathcal{H} \otimes \mathcal{H})$ and a quantum local channel $\mathcal{E}_{L}=\mathcal{E} \otimes \mathcal{I}$ the following inequality holds

$$
E\left(\mathcal{E}_{L}[\omega]\right) \leq E\left(\mathcal{E}_{L}\left[\Psi_{+}\right]\right),
$$

where $E$ is some (normalized) entanglement measure, i.e. $\max _{\omega} E(\omega)=E\left(\Psi_{+}\right)$, where $\Psi_{+}$is a maximally entangled state.

Proof. Let us assume the convexity of the entanglement measure, i.e. $E(\omega) \leq \sum_{j} q_{j} E\left(\omega_{j}\right)$. Under such assumption it is sufficient to consider only pure states, i.e. to show that $E\left(\mathcal{E}_{L}[\Phi]\right) \leq E\left(\mathcal{E}_{L}\left[\Psi_{+}\right]\right)$. Any pure bipartite state $\Phi=|\phi\rangle\langle\phi|$ can be written as $|\phi\rangle=I \otimes A\left|\psi_{+}\right\rangle$, where $A$ is a suitable linear operator. Hence we have $\mathcal{E}_{L}[\Phi]=(\mathcal{E} \otimes \mathcal{I})\left[(I \otimes A) \Psi_{+}\left(I \otimes A^{\dagger}\right)\right]=(I \otimes A)(\mathcal{E} \otimes$ $\mathcal{I})\left[\Psi_{+}\right]\left(I \otimes A^{\dagger}\right)=\mathcal{E}_{A} \mathcal{E}_{L}\left[\Psi_{+}\right]$. Next we will use the fact that local actions cannot increase the value of entanglement, i.e. $E\left(\mathcal{E}_{A}[\rho]\right) \leq E(\rho)$. Consequently, by putting $\rho=\mathcal{E}_{L}\left[\Psi_{+}\right]$we obtain the desired inequality $E\left(\mathcal{E}_{L}[\Phi]\right) \leq E\left(\mathcal{E}_{L}\left[\Psi_{+}\right]\right)$.

As a consequence we have that a local action applied on a set of maximally entangled states results in a set of states with the same amount of entanglement (irrespective of the measure we use). One can prove this property directly from the fact that all maximally entangled states are related by local unitary transformation applied on one subsystem, i.e. the states $\left|\Psi_{U}\right\rangle=I \otimes U\left|\Psi_{+}\right\rangle$form a set of all maximally entangled states. Applying the local operation $\mathcal{E} \otimes I\left[\Psi_{U}\right]=\mathcal{E} \otimes U\left[\Psi_{+}\right]=(\mathcal{I} \otimes U)\left(\mathcal{E} \otimes \mathcal{I}\left[\Psi_{+}\right]\right)=$ $(\mathcal{I} \otimes U)\left[\Omega_{\mathcal{E}}\right]$. This means that states $\Omega_{\mathcal{E}}=\mathcal{E} \otimes \mathcal{I}\left[\Psi_{+}\right]$ and $\Omega_{\mathcal{E}}^{U}=\mathcal{E} \otimes \mathcal{I}\left[\Psi_{U}\right]$ are locally unitary equivalent $\left(\Omega_{\mathcal{E}}^{U}=\mathcal{I} \otimes U\left[\Omega_{\mathcal{E}}\right]\right)$. Consequently, they contain the same amount of entanglement, which proves our statement. Such result can be extended to all states $\omega_{1}, \omega_{2}$ equivalent in the sense $\omega_{1}=\mathcal{I} \otimes U\left[\omega_{2}\right]$, for which the equality of entanglement is not affected by the local action $\mathcal{E} \otimes \mathcal{I}$.

The above theorem has served as the motivation to the main question considered in this paper. Specifically, whether all local channels of the form $\mathcal{E}_{L}=\mathcal{E} \otimes \mathcal{I}$ preserve the ordering induced by a given entanglement measure. Or in other words, for which entanglement measures $E$ the following implication holds

$$
E\left(\omega_{1}\right) \leq E\left(\omega_{2}\right) \Rightarrow E\left(\omega_{1}^{\prime}\right) \leq E\left(\omega_{2}^{\prime}\right),
$$

where $\omega_{j}^{\prime}=\mathcal{E} \otimes \mathcal{I}\left[\omega_{j}\right](j=1,2)$. One might think that for a "good" measure of entanglement such inequality should be valid. However, this property is not listed among the usually required properties of entanglement measures. Surprisingly enough, there is a simple argument that such "natural" property cannot hold in general. Firstly, we will present an explicit counterexample for the entanglement measure of two-qubit states called the concurrence [9]. Secondly, we will give a general argument why the answer to our question is negative, i.e. local actions may affect nontrivially the ordering induced by entanglement measures.

Example 1. Let us consider two families of two-qubit states: $i)$ Werner states $\varrho_{1}=q \Psi_{+}+(1-q) \frac{1}{4} I(0 \leq q \leq 1)$, and ii) pure states $\varrho_{2}=\Phi=|\phi\rangle\langle\phi|,| \phi\rangle=\alpha|00\rangle+\beta|11\rangle$ with $\alpha, \beta$ real and $\alpha^{2}+\beta^{2}=1$. Both of these families of states cover the whole interval of possible values of entanglement. Applying the local depolarizing channel $\mathcal{E}[\varrho]=p \varrho+(1-p) \frac{1}{2} I$ we obtain

$$
\begin{aligned}
& \varrho_{1}^{\text {in }} \stackrel{\stackrel{\mathcal{E} \otimes \mathcal{I}}{\longrightarrow} \varrho_{1}^{\text {out }}=p q \Psi_{+}+(1-p q) \frac{1}{4} I ;}{ } \\
& \varrho_{2}^{\text {in }} \stackrel{\mathcal{E} \otimes \mathcal{I}}{\longrightarrow} \varrho_{2}^{\text {out }}=p \Phi+(1-p) \frac{1}{2} I \otimes \varrho_{B},
\end{aligned}
$$

where $\varrho_{B}=\operatorname{Tr}_{A} \Phi$. The states $\left(\varrho_{1}^{\text {in }}, \varrho_{1}^{\text {out }}, \varrho_{2}^{\text {in }}, \varrho_{2}^{\text {out }}\right)$ belong to the set of states represented by $\varrho_{2}^{\text {out }}$, i.e. a mixture of pure state $\Phi$ with the mixed state $\frac{1}{2} I \otimes \varrho_{B}$. Therefore, it is sufficient to calculate the amount of entanglement for the state $\varrho_{2}^{\text {out }}$

$$
\varrho_{2}^{\text {out }}=\left(\begin{array}{cccc}
\alpha^{2} \frac{1+p}{2} & 0 & 0 & p \alpha \beta \\
0 & \beta^{2} \frac{1-p}{2} & 0 & 0 \\
0 & 0 & \alpha^{2} \frac{1-p}{2} & 0 \\
p \alpha \beta & 0 & 0 & \beta^{2} \frac{1+p}{2}
\end{array}\right) .
$$

The entanglement measure called the concurrence [9] is defined via the square roots of eigenvalues of the matrix $R=\varrho_{2}^{\text {out }}\left(\sigma_{y} \otimes \sigma_{y}\right)\left[\varrho_{2}^{\text {out }}\right]^{*}\left(\sigma_{y} \otimes \sigma_{y}\right)$. Let us denote by $\operatorname{Eig}[R]=\left\{\lambda_{1}, \lambda_{2}, \lambda_{3}, \lambda_{4}\right\}$ the eigenvalues of the matrix 


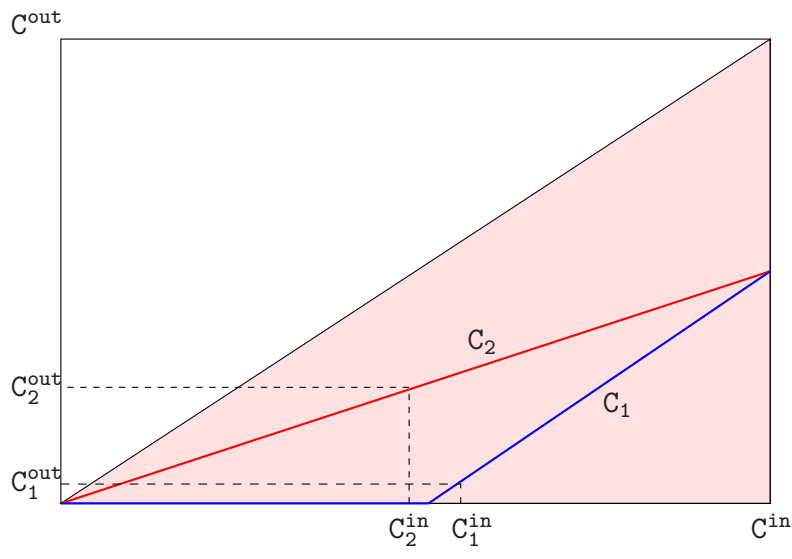

FIG. 1: (Color online) The input/output concurrence diagram for both families of states $\varrho_{1}, \varrho_{2}$ and for the depolarizing channel with $p=1 / 2$. The states from the counterexample 1 discussed in the paper are displayed and the change in the ordering is visible. The region under the line $C^{\text {out }}=C^{\text {in }}$ represents the allowed region that is achievable by local channels. Concurrence is measured in dimensionless units.

$R$ ordered in a decreasing order, i.e. $\lambda_{1} \geq \lambda_{2} \geq \lambda_{3} \geq$ $\lambda_{4}$. The concurrence is given by the expression $C=$ $\max \left\{0, \sqrt{\lambda_{1}}-\sqrt{\lambda_{2}}-\sqrt{\lambda_{3}}-\sqrt{\lambda_{4}}\right\}$. For the states $\varrho_{2}^{\text {out }}$ one can find a compact expression for the concurrence $C_{2}^{\text {out }}=$ $\alpha \beta(3 p-1)$. Using this formula we find all quantities that are need for our considerations. In particular,

$$
\begin{array}{cc}
C_{1}^{\text {in }}=\frac{1}{2}(3 q-1) & \stackrel{\mathcal{E} \otimes \mathcal{I}}{\longrightarrow} C_{1}^{\text {out }}=\frac{1}{2}(3 p q-1) ; \\
C_{2}^{\text {in }}=2 \alpha \beta & \stackrel{\mathcal{E} \otimes \mathcal{I}}{\longrightarrow} C_{2}^{\text {out }}=\alpha \beta(3 p-1) .
\end{array}
$$

Moreover, we are able to determine the functional dependence $C^{\text {out }}=f\left(C^{\text {in }}\right)$. In particular, we obtain parametric linear functions

$$
\begin{aligned}
& \varrho_{1}: C_{1}^{\text {out }}=\max \left\{0, p C_{1}^{\text {in }}+\frac{1}{2}(p-1)\right\} \\
& \varrho_{2}: C_{2}^{\text {out }}=\max \left\{0, \frac{3 p-1}{2} C_{2}^{\text {in }}\right\}
\end{aligned}
$$

where the parameter $p$ represents the action (strength) of the depolarizing channel. Let us consider the depolarizing channel with $p=0.5$ and two input states such that $C_{1}^{\text {in }}=\frac{1}{2}+\epsilon, C_{2}^{\text {in }}=\frac{1}{2}-\epsilon$. The degree of entanglement of the resulting output states reads $C_{1}^{\text {out }}=\frac{1}{2} \epsilon$ and $C_{2}^{\text {out }}=\frac{1}{8}-\frac{1}{4} \epsilon$. It is easy to see that whenever $0<\epsilon<\frac{1}{6}$ the original inequality $C_{1}^{\text {in }}>C_{2}^{\text {in }}$ is transformed into the opposite inequality, i.e. $C_{1}^{\text {out }}<C_{2}^{\text {out }}$ [see Fig. 1].

Example 2. Let us assume a four-qubit system divided into two pairs: the left $(\mathrm{L})$ and the right $(\mathrm{R})$. Consider two pure states $\left|\Omega_{j}\right\rangle=\left|\psi_{j}\right\rangle_{L_{1} R_{1}} \otimes\left|\phi_{j}\right\rangle_{L_{2} R_{2}}$ for $j=1,2$, i.e. the parties $\mathrm{L}$ and $\mathrm{R}$ share two pairs of two-qubit pure states. We will act locally on the right couple of qubits only, i.e. we apply the channel $\mathcal{E}=\mathcal{I}_{L_{1} L_{2}} \otimes \mathcal{E}_{R_{1} R_{2}}$. In particular, we assume that $\mathcal{E}_{R_{1} R_{2}}=\mathcal{E} \otimes \mathcal{I}$ and $\mathcal{E}$ is the contraction into a fixed pure state, i.e. $\varrho \rightarrow \Xi$. The additivity of entanglement for pure states implies that initially $E\left(\Omega_{1}\right)=E\left(\Psi_{1}\right)+E\left(\Phi_{1}\right)=x_{1}+y_{1}$ and $E\left(\Omega_{2}\right)=$ $E\left(\Psi_{2}\right)+E\left(\Phi_{2}\right)=x_{2}+y_{2}$. After the action of the local channel we obtain the states $\Omega_{1}^{\prime}=\left[\operatorname{Tr}_{R_{1}} \Psi_{1}\right] \otimes \Xi_{R_{1}} \otimes \Phi_{1}$ and $\Omega_{2}^{\prime}=\left[\operatorname{Tr}_{R_{1}} \Psi_{2}\right] \otimes \Xi_{R_{1}} \otimes \Phi_{2}$. The convexity implies $E\left(\Omega_{1}^{\prime}\right) \leq \lambda E\left(\left|\omega_{1}\right\rangle\left\langle\omega_{1}\right| \otimes \Xi \otimes \Phi_{1}\right)+(1-\lambda) E\left(\left|\omega_{2}\right\rangle\left\langle\omega_{2}\right| \otimes \Xi \otimes\right.$ $\left.\Psi_{1}\right)$, where $\left|\omega_{j}\right\rangle$ are eigenvectors of $\operatorname{Tr}_{R_{1}} \Psi_{1}$ and $\lambda, 1-\lambda$ are the corresponding eigenvalues. On the right hand side we have pure states, for which the entanglement can be found by using the additivity of $E$ for pure states. We obtain $E\left(\Omega_{1}^{\prime}\right) \leq \lambda E\left(\Phi_{1}\right)+(1-\lambda) E\left(\Phi_{1}\right)=E\left(\Phi_{1}\right)=$ $y_{1}$ and by a similar line of arguments we find $E\left(\Omega_{2}^{\prime}\right) \leq$ $y_{2}$. Let us consider that originally $x_{1}+y_{1}>x_{2}+y_{2}$ $\left(E\left(\Omega_{1}\right)>E\left(\Omega_{2}\right)\right)$ and $y_{2}>y_{1}=0$. After the action of the local channel we have $E\left(\Omega_{1}^{\prime}\right)=0$ and $E\left(\Omega_{2}^{\prime}\right) \leq y_{2}$, i.e. we obtain an un-sharp inequality $E\left(\Omega_{1}^{\prime}\right) \leq E\left(\Omega_{2}^{\prime}\right)$. In what follows we will argue that this inequality cannot be saturated, i.e. $0<E\left(\Omega_{2}^{\prime}\right)$. In other words, we would like to show that there is still some entanglement present between the left and the right systems. Since one pair $\left(\Phi_{1}\right.$, or $\left.\Phi_{2}\right)$ has not been affected at all by the action of the local channel it can be still used to violate Bell inequalities (the state $\Phi_{2}$ is pure and entangled). This violation of Bell inequalities is an evidence of presence of entanglement between the two parties. In conclusion, $E\left(\Omega_{1}^{\prime}\right)<E\left(\Omega_{2}^{\prime}\right)$, but $E\left(\Omega_{1}\right)>E\left(\Omega_{2}\right)$.

Let us summarize the line of arguments in the last example: We have two pairs of qubits such that $x_{1}+$ $y_{1}>x_{2}+y_{2}$ and we put $y_{1}=x_{2}=0$, i.e. $x_{1}>$ $y_{2}$. In other words we have two different pure states of two pairs: 1) the entangled $\otimes$ factorized, and 2) the factorized $\otimes$ entangled states. The contractive channel is applied on the first pair only. The first state is transformed and become unentangled, while the second state can be used to violate Bell inequalities, because the entangled part has not been affected at all by the action of the local channel. As a result, we conclude that the entanglement-induced ordering of states is not absolute under the action of local channels. Consequently, in some circumstances less entangled states can be more "robust" against local operations.

Using the above theorem we can justify the choice of maximally entangled states in our analysis of timedependent entanglement ordering induced by local evolutions. That is, maximally entangled states when subjected to a local evolution (i.e., one particle is evolving freely while the second is subjected to an action of a local channel) bound the maximally available entanglement. However, one has to be careful to draw general conclusions about the entanglement behavior. Here we have analyzed the simplest situation in which only one of the subsystems undergone a nontrivial local evolution. Let us note that the Theorem does not hold if one considers bi-local channels, i.e. in that case even the maximality of entanglement is a "relative" notion.

The second example illustrates that the entanglementinduced ordering is not absolute in general: The original 
entanglement-based ordering (irrespective of the choice of the entanglement measure) within a set of states can be changed (even) under the action of a local channel. However, for two-qubit systems it is still an open question, whether there exist an entanglement measure, for which the entanglement-induced state ordering is not affected by the action of local channels. Another open problem is the characterization of those local channels that preserve the state ordering induced by a given measure, for instance the concurrence. The characterization and classification of channels using the $E^{\text {out }} v s$. $E^{\text {in }}$ diagram can be very useful in such analysis. This approach can be applied to any measure and any channel. For instance, the action of local unitary channels $U \otimes \mathcal{I}$ are represented by the line $E^{\text {out }}=E^{\text {in }}$ in this diagram. Analogously, for entanglement-breaking channels [20, 21] we have $E^{\text {out }}=0$, i.e. the action of these channels is represented again by a line in the $E^{\text {out }}$ vs $E^{\text {in }}$ diagram. Both of these families of channels preserve the entanglementinduced ordering for an arbitrary measure of entanglement. Presently it is still an open problem whether there exist some other local channels that have the same property (at least for a particular measure of entanglement).

From the construction that has been used in Example 1 it follows that whenever we obtain a region that is bounded by two lines corresponding to two input states that exhibit the same degree of initial entanglement in the $E^{\text {out }}$ vs $E^{\text {in }}$ diagram we can find two states for which the entanglement-induced ordering is not preserved. Channels represented by a line in $E^{\text {out }} v s . E^{\text {in }}$ diagram not only preserve the state ordering, but they also preserve the "isoentangled" classes of states. In other words, if the "in" states are equally entangled, then the "out" states are equally entangled as well. This is a very confining constraint imposed on the action of local quantum channels and therefore we do not expect that there exists a nontrivial channel (except unitary, or entanglement-breaking channels mentioned above) that preserve the entanglement-induced ordering.
In conclusion, we have addressed the question how the entanglement between two components of a bipartite system behaves under the action of local channels of the form $\mathcal{E} \otimes \mathcal{I}$. We have shown that a set of maximally entangled states is by the action of the local channel $\mathcal{E} \otimes \mathcal{I}$ transformed into the set of states that exhibit the same degree of entanglement. Moreover, this degree represents an upper bound of entanglement that can be available at the output of the channel irrespective what is the input state. We have shown that within this bound the the entanglement-induced state ordering is "relative" and can be changed by the action of local channels. Moreover, our study suggests that this is a rather common property of most of the local channels. That is, most of the local channels affect the entanglement-induced state ordering. Our analysis opens several interesting questions related to "dynamics" of entanglement and provides a novel tool of the characterization of arbitrary quantum operations (both local and global) using the $E^{\text {out }} v s . E^{\text {in }}$ diagram. Let us note that already for the so called bilocal channels of the form $\mathcal{E}_{1} \otimes \mathcal{E}_{2}$ this picture looks differently, because in this case even the set of maximally of entangled states transforms differently, i.e. the output states might not posses the same degree of entanglement. In addition, it is not clear whether the "maximality" of entanglement is absolute, or not, i.e. whether $\omega_{\max }=\arg \sup _{\omega} E\left(\mathcal{E}_{1} \otimes \mathcal{E}_{2}[\omega]\right)$ is one of the maximally entangled states, or not. This problem illustrates the fact that dynamical features of quantum entanglement are still not understand in all details and many interesting features remain to be explored and understood.

\section{Acknowledgments}

This work has been supported partially by European projects QGATES, CONQUEST, by Slovak project APVT and by GAČR GA201/01/0413. We also acknowledge the support of Slovak Academy of Sciences via the project CE-PI (I/2/2005) and VB would like to thank to the Alexander von Humboldt Foundation.
[1] M.A. Nielsen and I.L. Chuang, Quantum Computation and Quantum Information, (CUP, Cambridge, 2000)

[2] J. Preskill, Quantum theory of Information and Computation, www.theory.caltech.edu/people/preskill

[3] E. Schroedinger, Proc. Cambridge Philos. Soc. 31, 555 (1935)

[4] V. Coffman, J. Kundu, and W.K. Wootters, Distributed entanglement, Phys. Rev. A 61, 052306 (2000)

[5] T.J. Osborne and F. Verstraete, General Monogamy Inequality for Bipartite Qubit Entanglement, Los Alamos e-print arXive quant-ph/0502176

[6] V. Vedral, High Temperature Macroscopic Entanglement, New J. Phys. 6, 102 (2004)

[7] M. Plenio and V. Vedral, Entanglement in Quantum Information Theory, Contemp. Phys. 39, 431-466 (1998)

[8] R.F. Werner, Quantum states with Einstein-PodolskiRosen correlations admitting a hidden-variable model,
Phys. Rev. A 40, 4277-4281 (1989)

[9] W.K. Wootters, Entanglement of formation for an arbitrary state of two qubits, Phys. Rev. Lett. 80, 2245 (1998)

[10] L. Henderson and V. Vedral, Information, Relative Entropy of Entanglement and Irreversibility, Phys. Rev. Lett. 84, 2263-2266 (2000)

[11] J. Eisert and M.B. Plenio, A comparison of entanglement measures, J. Mod. Opt. 46, 145 (1999)

[12] A. Miranowicz and A. Grudka, Ordering two-qubit states with concurrence and negativity, Phys. Rev. A 70, 032326 (2004)

[13] K. Zyckowski, P. Horodecki, M. Horodecki, and R. Horodecki, Dynamics of quantum entanglement, Phys. Rev. A 65, 012101 (2001),

[14] F. Verstraete, K. Audenaert, T. De Bie, and B. De Moor, Maximally entangled states of two qubits, Phys. Rev. A 64, 012316 (2001), 
[15] J.M. Cai, Z.W. Zhou, and G.C. Guo, Stability of pairwise entanglement in a decoherent environment, LANL preprint archive quant-ph/0507021

[16] K. Roszak and P. Machnikowski, Complete disentanglement by partial pure dephasing, LANL preprint archive quant-ph/0507028

[17] E.S. Cardoso, M.C. de Oliveira, and F. Furuya, Entanglement versus mixedness for coupled qubits under a phase damping channel, LANL preprint archive quant$\mathrm{ph} / 0507212$

[18] X.T. Liang, Initial decoherence and disentanglement of open two-qubit systems, LANL preprint archive quant$\mathrm{ph} / 0507226$

[19] M. Ziman and V. Bužek, Purity vs. concurrence picture under subsystems evolution, LANL preprint archive quant-ph/0508106

[20] The channel is called as the entanglement-breaking if the state $\varrho^{\prime}=\mathcal{E} \otimes \mathcal{I}[\varrho]$ is separable for all $\varrho$.

[21] M. Horodecki, P.W. Shor, and M.B. Ruskai, General entanglement-breaking channels, Rev. Math. Phys 15, 629-641 (2003) 